\title{
ECONOMETRIA: ISTANZE DELL'ECONOMIA E PARALLELISMI CON LE SCIENZE NATURALI
}

\author{
MARIO FALIVA (*) \\ MARIA GRAZIA ZOIA (*)
}

RiASSUNTO. - Il saggio, dopo aver operato un raffronto fra le linee guida dell'econometria classica e dell'econometria delle serie storiche, rivolge l'attenzione ai rapporti che collegano, mediante un ponte ideale, analisi econometrica e metodo scientifico.

Lo studio della struttura causale dei modelli macroeconometrici con la teoria dei sistemi, da un lato, ed il problema della enucleazione delle componenti dalle serie storiche con la teoria dei filtri dall'altro, costituiscono due esempi emblematici della proficua interazione fra problemi e metodi propri dell'econometria e tecniche delle scienze naturali.

$* * *$

ABSTRACT. - The paper, after casting a glance at the facing heads of classical and timeseries econometrics, turns the spotlight on the ideal bridge linking econometric analysis to scientific method.

The investigation into the causal structure of macroeconometric models within a system-theory framework, on one hand, and the detection of components from economic time series by filtering techniques on the other, provide two emblematic examples of the fruitful melting pot of econometric and natural-science methodologies.

(*) Università Cattolica del Sacro Cuore, Milano. 


\section{LA NOZIONE DI ECONOMETRIA AI SENSI DELLO STATUTO DELL'"ECONOMETRIC SOCIETY"}

L'econometria nasce con la costituzione, agli albori degli anni trenta, dell'Econometric Society il cui Statuto recita:

"La società Econometrica è una associazione internazionale che si propone di favorire il progresso della teoria economica nei suoi collegamenti con la statistica e la matematica....

Il suo scopo primario è quello di promuovere quegli studi che mirino ad unificare l'approccio teorico-quantitativo con quello empirico-quantitativo nell'analisi dei fenomeni economici, studi che siano ispirati da un pensiero costruttivo e rigoroso simile a quello che è proprio delle scienze naturali.....".

\section{L'ECONOMETRIA CLASSICA: LE IDEE GUIDA}

In un'accezione classica si può guardare all'econometria come a quella branca delle discipline economiche in cui gli strumenti della teoria economica, della matematica e della statistica vengono applicati all'analisi dei fenomeni economici.

L'obiettivo di affrontare l'analisi dei fenomeni economici con una forma mentis simile a quella che domina nell'ambito delle scienze naturali, è la chiave di volta per una simbiosi proficua fra conoscenze teorico-speculative - che si traducono in un insieme di ipotesi a priori esplicitamente formulate -, ed un complesso di indicazioni - a supporto o parziale rettifica delle ipotesi suddette - desumibili, con un adeguato grado di plausibilità, alla luce dell'evidenza empirica dei dati.

Possiamo utilmente avvalerci di un'immagine di econometria come amalgama di teoria economica, matematica, statistica ed evidenza empirica, e raffigurarla con un diagramma. 


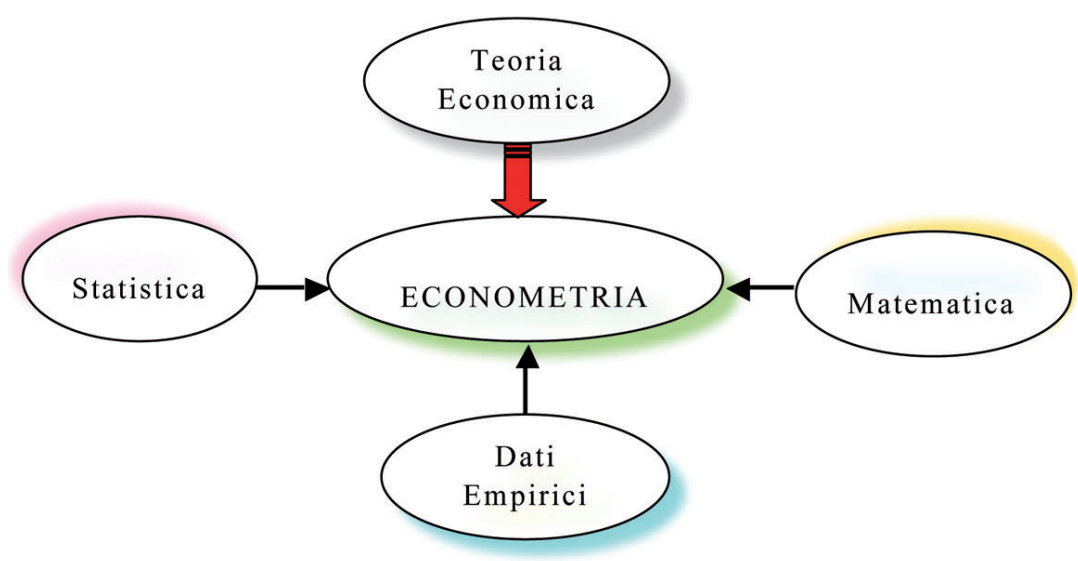

Compito della teoria economica è quello di indicare le variabili rilevanti (endogene ed esogene) per la spiegazione del fenomeno allo studio, nonchè le caratteristiche delle relazioni che le legano, da cui far scaturire la formulazione del modello econometrico. I dati empirici forniscono il supporto statistico con cui operare la quantificazione delle relazioni del modello.

L'econometria così intesa mira all'obiettivo di confrontare la teoria economica con i dati storici, di verificare e/o suggerire l'esistenza di relazioni ipotetiche tra le variabili economiche, di precisare la forma di dette relazioni e quantificarle. L'econometria in questa accezione può contribuire al progresso dell'economia come scienza.

L'impalcatura dell'econometrica classica poggia crucialmente sulla nozione di modello.

Un modello econometrico è una rappresentazione formalizzata della teoria economica ed assume tipicamente la configurazione di un sistema di equazioni parametriche. Dette equazioni esprimono le relazioni che intercorrono tra le variabili (endogene ed esogene) ai sensi della teoria economica

Alle equazioni del modello si può dare una chiave di lettura come relazioni causa-effetto ovvero come relazioni affette da meccanismi di feedback che sono all'origine della simultaneità.

I modelli econometrici hanno natura dinamica e si configurano conseguentemente come sistemi di equazioni alle differenze. 
L'incompletezza della teoria economica e l'esigenza di parsimonia che accompagna il processo di specificazione conferiscono natura stocastica alle equazioni del modello, con la presenza di termini d'errore interpretabili come variabili casuali con funzioni compensative per le discrepanze fra modello e realtà oggetto di studio.

In sintesi un modello econometrico si configura come un sistema di equazioni dinamiche, stocastiche, espresse in forma parametrica (con possibili non-linearità nei parametri e/o nelle variabili).

Una ricerca econometria si articola in una sequenza di fasi caratteristiche come delineato nel diagramma a blocchi sottostante.

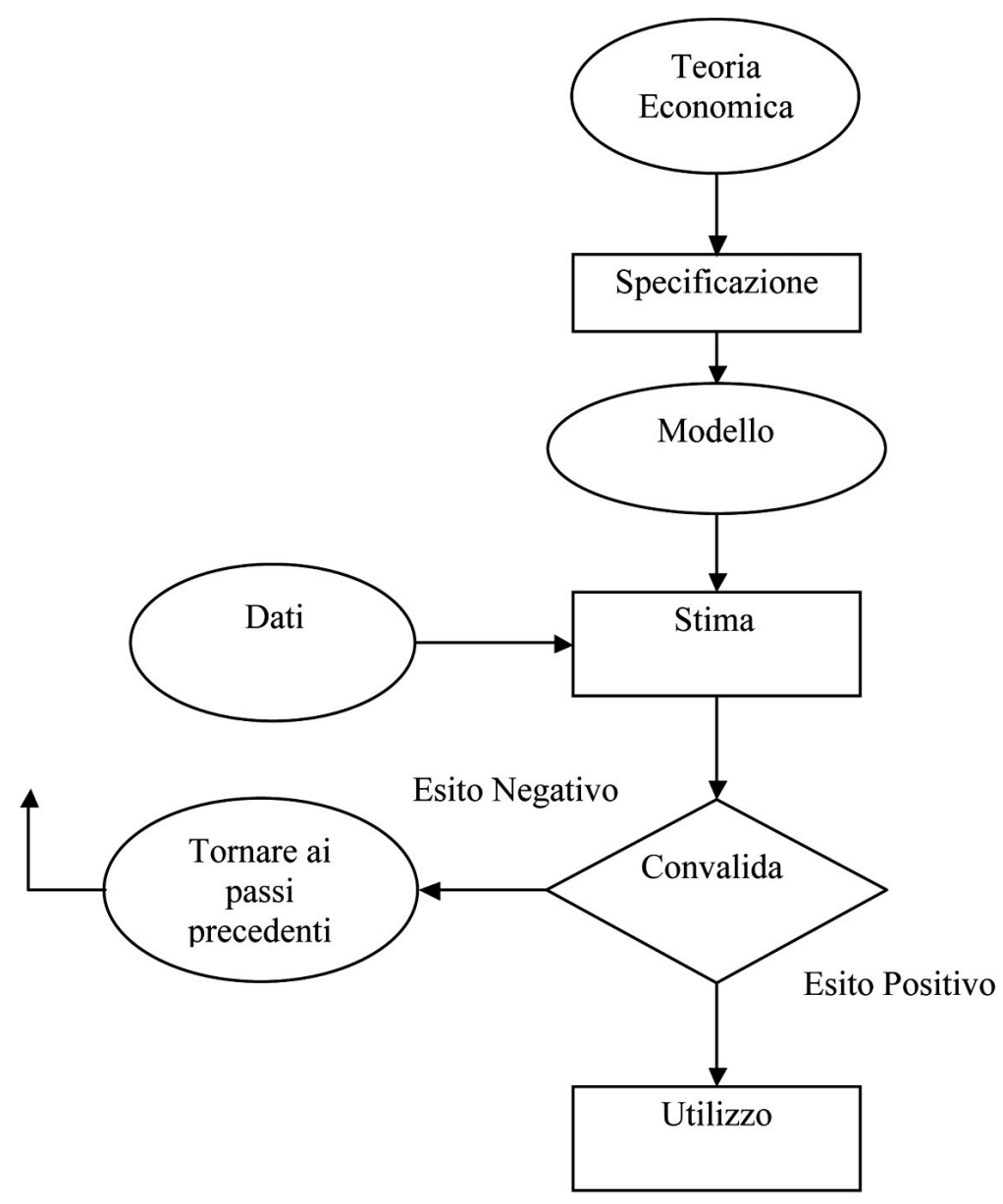




\section{L'ECONOMETRIA DELLE SERIE STORICHE: LE IDEE GUIDA}

L'econometria classica ha la sua immagine speculare (e complementare) nella cosiddetta econometria delle serie storiche. Nell'econometria delle serie storiche, l'assunto - proprio dell'econometria classica - del ruolo determinante svolto dalla teoria economica nella specificazione del modello è confutato, ed il nucleo della modellizzazione econometrica poggia crucialmente su una specificazione VAR (modello autoregressivo vettoriale, ovvero multivariato), col riflesso dell'analisi di integrazione (e cointegrazione) volta a recepire la natura non stazionaria delle variabili economiche.

Ciò comporta che lo schema concettuale imperniato sull'interazione fra teoria economica, matematica, statistica ed evidenza empirica, che connota l'econometria classica, trovi una reinterpretazione in chiave speculare nell'econometria delle serie storiche, traducendosi in una sorta di ribaltamento di ruoli fra peso specifico della evidenza empirica e ruolo guida della teoria economica, come illustra la figura sottostante.

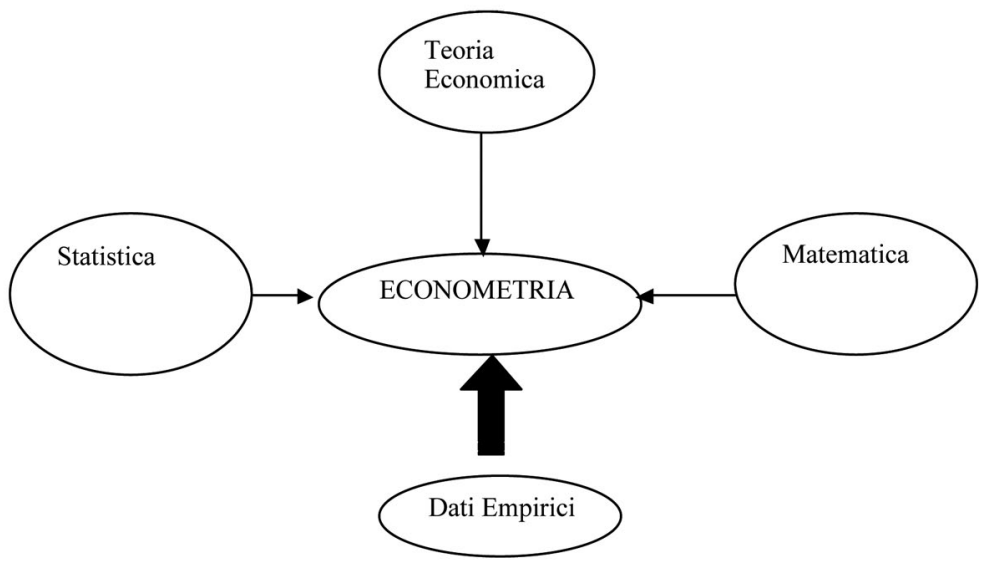

Così, quasi come un Giano bifronte, mentre nella econometria classica l'evidenza empirica gioca un ruolo di validazione della teoria economica, nell'econometria delle serie storiche sono i dati a definire i contorni del quadro di riferimento e la teoria economica interviene per certificare la coerenza dei risultati acquisiti su base empirica con i principi condivisi dagli economisti.

Il modus operandi dell'econometria delle serie storiche è delinea- 
to dallo schema a blocchi sottostante, che offre l'immagine dell'altra faccia dell'econometria.

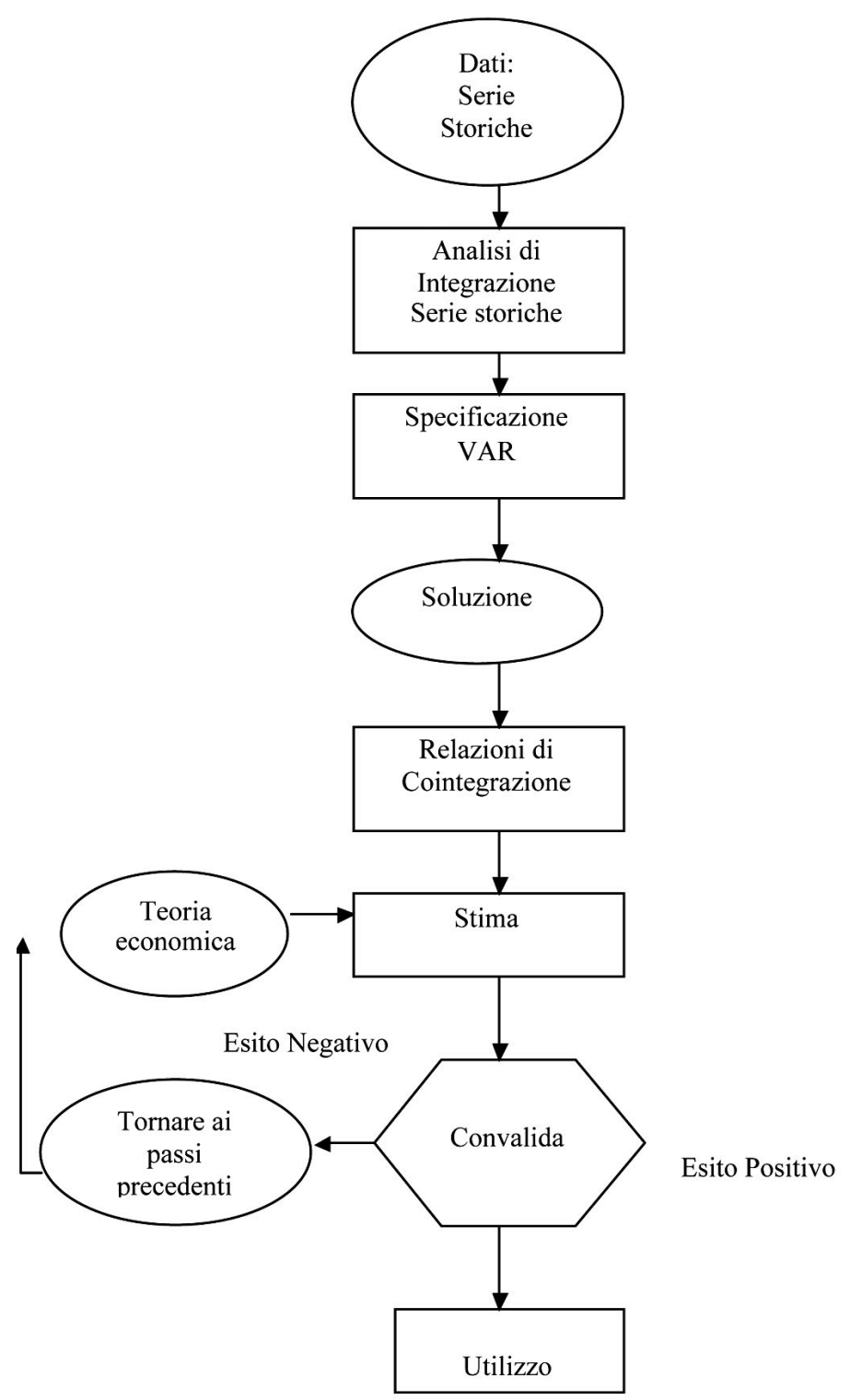


Come casi emblematici di simbiosi fra le problematiche inerenti alle ricerche econometriche e strumenti e metodi sviluppati nell'ambito delle scienze fisiche e naturali si possono ricordare i seguenti:

a) L'analisi della struttura causale del modello nell'ottica della teoria dei sistemi ad anello aperto e chiuso, quali immagini speculari delle nozioni di ricorsività ed interdipendenza.

b) L'enucleazione dalle serie storiche economiche - che costituiscono la base empirica di una ricerca econometrica - delle componenti di lungo e medio periodo oggetto della modellizzazione, identificabili con le nozioni di trend e ciclo.

Poiché alla qualificazione di lungo, medio e breve periodo per le componenti delle serie storiche economiche, può essere fatta corrispondere - nel parallelo dominio frequenziale - una caratterizzazione per bande di frequenze (basse, intermedie ed alte), l'enucleazione delle componenti dai dati può essere opportunamente condotta con le tecniche proprie della teoria dei segnali e dei filtri (con le naturali analogie elettriche ed ottiche e con le pertinenti strumentazioni analitiche).

Un ulteriore punto di raccordo è rappresentato dalle componenti stocastiche in gioco, l'errore dei modelli strutturali è comunemente assimilato alla nozione di rumore bianco (ovvero colorato in presenza di autocorrelazione), laddove i cosiddetti trend stocastici dell'econometria delle serie storiche si identificano con la nozione di cammino casuale.

Nei prossimi due paragrafi ci proponiamo di dare un contenuto formale ai temi di cui abbiamo fatto cenno.

\section{L'ANALISI DELLA STRUTTURA CAUSALE DEI MODELLI ECONOMETRICI NELL'OTTICA DELLA TEORIA DEI SISTEMI}

In un modello econometrico ogni singola equazione rappresenta una specifica relazione, desunta dalla teoria economica, che esprime un legame fra una variabile endogena ( $y$, al primo membro) ed un insieme di variabili (altre endogene contemporanee e predeterminate $z$, al secondo membro) che sono considerate esplicative della prima.

Le relazioni espresse dal modello possono rappresentare dei legami unidirezionali, interpretabili in termini di causa-effetto. La natura delle connessioni fra variabili può peraltro condurre a confi- 
gurare l'esistenza di legami bidirezionali, caratterizzati cioè dalla presenza di fenomeni di feedback che si traducono nel fenomeno della interdipendenza.

Il collegamento fra i concetti di causalità e interdipendenza e le nozioni di modello come sistema ad anello aperto e chiuso, consente di applicare all'analisi della struttura causale dei modelli econometrici strumenti e tecniche messi a punto in ingegneria per lo studio dei sistemi con retro-azione.

Come è noto un modello econometrico - che supporremo lineare per comodità espositiva - può essere rappresentato in forma strutturale come una equazione del tipo:

$$
y=\Gamma y+A z+\varepsilon
$$

dove $\Gamma$ ed A rappresentano matrici di coefficienti, y e $z$ stanno ad indicare $\mathrm{i}$ vettori delle endogene e delle predeterminate, e $\varepsilon$ è un vettore di errori non sistematici.

Ignorando il termine di errore, l'equazione suddetta può essere così riformulata:

$$
y=\left[\begin{array}{ll}
I, & A
\end{array}\right]\left[\begin{array}{l}
0 \\
z
\end{array}\right]+\left[\begin{array}{ll}
I, & A
\end{array}\right]\left[\begin{array}{l}
\Gamma \\
0
\end{array}\right] y
$$

e quindi letta come "forma primaria" di un sistema ad anello chiuso come rappresentato nella figura che segue.

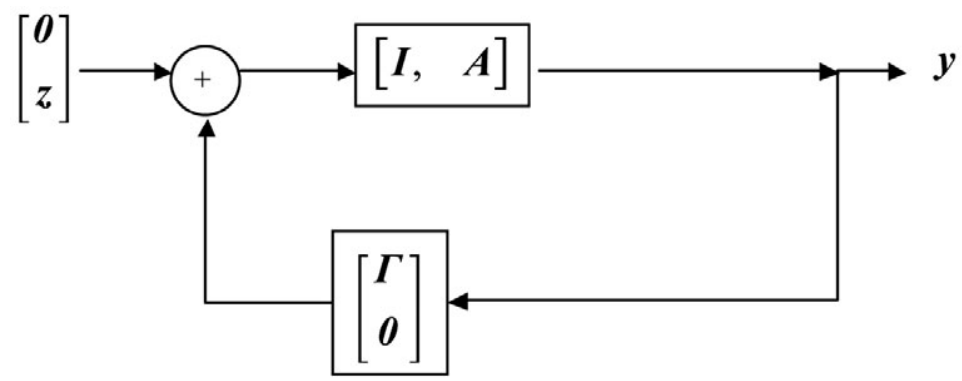

Alla luce della rappresentazione di cui sopra la matrice 


$$
\left[\begin{array}{ll}
I, & A
\end{array}\right]\left[\begin{array}{l}
\Gamma \\
0
\end{array}\right]=\Gamma
$$

assume il significato di fattore di reazione del sistema.

La natura causale o interdipendente dei legami fra le endogene, risulta dipendere dalle caratteristiche strutturali della matrice di reazione $\Gamma$, ai sensi delle seguenti proposizioni:

a) se $\Gamma$ è nulla il sistema è ad anello aperto: è il caso di un modello con equazioni non interconnesse;

b) se tutti gli autovalori di $\Gamma$ sono nulli il modello è del tipo a catena causale: è il caso di un sistema ricorsivo;

c) se $\Gamma$ ha struttura triangolare a blocchi il modello si compone di sottomodelli connessi in cascata: è il caso di un sistema ricorsivo a blocchi;

d) se $\Gamma$ è irriducibile il modello è interdipendente.

\section{L'ENUCLEAZIONE DAI DATI STORICI DELLE COMPONENTI DI INTERESSE NELL'OTTICA DELLA TEORIA DEI SEGNALI}

Le serie storiche economiche $\left(x_{t}\right)$ - con cadenza tipicamente mensile o trimestrale - costituiscono il materiale empirico di base delle ricerche econometriche.

Dette serie si presentano come la risultante dell'effetto congiunto di componenti comunemente note come trend $\left(f_{t}\right)$, ciclo $\left(c_{t}\right)$, stagionalità $\left(s_{t}\right)$ e componente accidentale $\left(\eta_{t}\right)$

Il significato delle varie componenti può essere sintetizzato come segue:

- la nozione di trend $\left(f_{t}\right)$ va identificata con il comportamento regolare di fondo, o di lungo periodo, di una serie ed è caratterizzato da un andamento monotonico o unimodale sull'arco temporale considerato.

- La nozione di ciclo $\left(c_{t}\right)$ è quella di componente legata ai movimenti congiunturali, o di medio periodo, della serie. Tale componente presenta un andamento oscillatorio alquanto irregolare, il cui periodo è dell'ordine di alcuni anni con un'ampiezza delle escursioni che può mutare nel tempo (ciclo evolutivo, $c_{t}^{(e v)}$ ). 
- La nozione di componente stagionale $\left(s_{t}\right)$ è quella di fluttuazione della serie legata al ritmo delle stagioni, e come tale riferibile ai movimenti di breve periodo. Detta componente presenta un andamento oscillatorio regolare di periodicità annuale, con ampiezza variabile nel tempo (stagionalità evolutiva, $s_{t}^{(e v)}$ ).

La componente accidentale $\left(\eta_{t}\right)$ va intesa come l'effetto risultante dalle fluttuazioni erratiche che influenzano in maniera non sistematica il fenomeno in esame.

Un modello di composizione semplice ed efficace al tempo stesso è il seguente:

$$
x_{t}=p_{t}+s_{t}^{(e v)}+\eta_{t}
$$

dove

$$
p_{t}=f_{t}+c_{t}^{(e v)}
$$

é la componente ciclo-trend e l'evolutività è assimilata ad un meccanismo interpretabile come modulazione di ampiezza (nella terminologia della teoria dei segnali) ad opera delle componenti di più lunga durata.

Trend, ciclo e stagionalità, congiuntamente presi, costituiscono la componente sistematica, interpretabile come segnale, della serie mentre il termine residuale è una componente di disturbo, interpretabile come rumore.

E' possibile dare delle componenti all'oggetto una chiave di lettura in termini frequenziali, facendo corrispondere alle nozioni di lungo, medio e breve periodo le nozioni di basse, medie ed alte frequenze.

Così facendo, la componente ciclo-trend si colloca nella gamma delle frequenze medio-basse, mentre la componente stagionale si colloca alle alte frequenze (la componente di disturbo si spalma sull'intero arco delle frequenze ammissibili). 


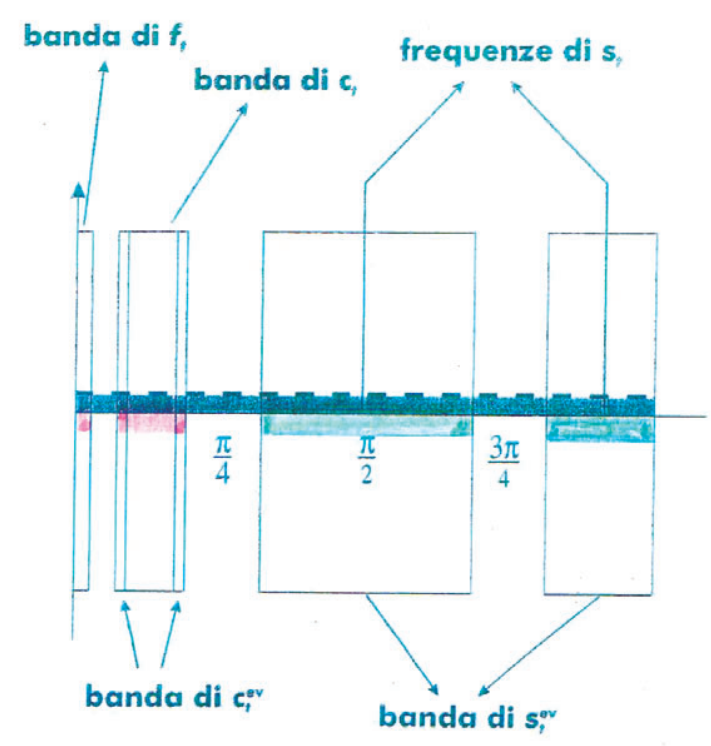

- - - -

arco di frequenze di pertinenza della componente erratica

In quest'ottica, il problema dell'enucleazione (ovvero della stima) del ciclo-trend assume le connotazioni proprie di un problema di filtraggio, ovvero della ricerca di una trasformazione che, operando sulla serie originale alla stregua di un filtro passa-basso, lasci inalterate le componenti di bassa frequenza, non attenui significativamente le componenti di media frequenza ed elimini, o comunque attenui drasticamente, le componenti di alta frequenza.

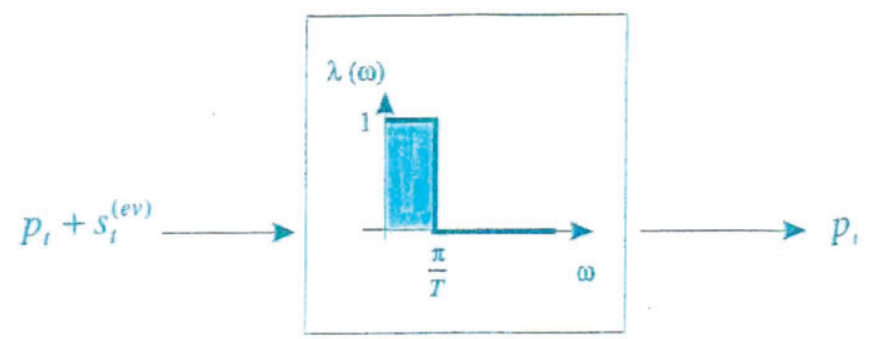

Azione del filtro ideale passo-basso sulle componenti sistematiche della serie. 


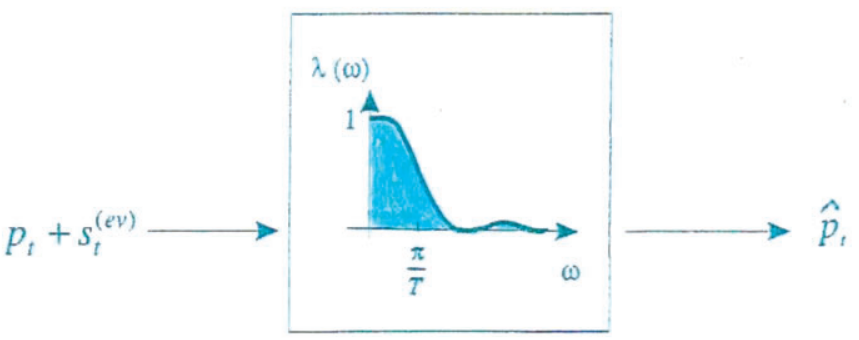

Azione di un filtro passo-basso effettivo sulle componenti sistematiche della serie.

\section{BIBLIOGRAFIA}

M. FALIVA, Econometria. Principi e Metodi. UTET, Torino, 1987.

M. FALIVA, L'analisi dei modelli econometrici nell'ambito della teoria dei sistemi, in Il Ruolo dell'Econometria nell'Ambito delle Scienze Economiche (a cura di M.Faliva). il Mulino, Bologna, 1991.

M. FALIVA e M.G.ZOIA, Topics in Dynamic Model Analysis. Springer, Berlino, 2006.

M. G. ZOIA, La nozione di spettro stilizzato ed il suo ruolo nell'analisi delle serie storiche economiche. Statistica, LVI, 1996, pp. 103-127.

M. G. ZOIA, Processi AR e processi integrati: dal caso univariato alla modellistica econometrica. Rivista Internazionale di Scienze Sociali, CVII, 1999, pp. 31-47. 